matters arising

\section{Assessment of risk of sudden death in infants}

THE study by Carpenter and Emery ${ }^{\natural}$ into the incidence of sudden death in children raises several methodological questions. They have clearly demonstrated that their classification into high- and low-risk groups works, but their evaluation of surveillance is less convincing.

Carpenter and Emery set out to assign children randomly to observed and control groups, yet children born at certain periods (holidays) were all assigned to control. While there may be no reason to suppose that these children are different from others, there is equally no reason to suppose that they are not. They should have been excluded from the analysis altogether if they could not be assigned to the observed group.

In addition, Carpenter and Emery failed to demonstrate a significant difference between observed and control groups. This is not surprising, as such failure was built in to the design of the study. If the proportion of deaths in that 'at risk' population were that found in the control group, 9.8/1,000, and the treatment reduced this to $1 / 1,000$, then the chance of finding a significant difference at the $5 \%$ level would be $70 \%$ (ref. 2). If the mortality were reduced to $3 / 1,000$, as in the sample, the chance of finding a significant difference would be less than $50 \%$. Even if the treatment were effective, it would be very unlikely it could be demonstrated.

Having failed, for the above if for no other reason, to demonstrate a significant difference between observed and treated groups, Carpenter and Emery combine those who were assigned to treatment but who refused it with the control group. There is no reason to suppose that those who refused are comparable to those who accepted, indeed, it would seem unlikely that they are. The refusers should be combined with the observed if we are to be true to the intention of the randomised trial. This gives a death rate of $6.0 / 1,000$ among the observed group to compare to $9.8 / 1,000$ in control, a difference which could easily have arisen by chance.

There seems to be no. justification for any service innovation in this study. However, the findings so far are certainly worth pursuing, and reanalysis as suggested, combined with a further study period involving more children, would answer the question one way or the other.

St George's Hospital,

$$
\text { J. M. Bland }
$$

Medical School, Department

of Clinical Epidemiology and

Social Medicine,

Cranmer Terrace,

London SW17, UK 1. Carpenter, R. G. \& Emery, J. L. Nature 268, 724-
725 (1977).
2. Snedecor, G. W. \& Cochran, W. G. Statistical
Methods, 6th ed. 221-222 (Iowa. State University
Press, 1967).

Carpenter and EMery RePly-Bland ${ }^{1}$ raises several points requiring comment. His conclusion that "there seems to be no justification for any service innovation in this study" arises from applying the theory of hypothesis testing to a decision problem. Using his approach, one starts with the most obvious null hypothesis, regardless of how implausible it may be, and acts on the alternative hypothesis only when the null hypothesis is rejected, irrespective of the consequences of a wrong decision. It is a simplistic approach to statistics that is widely taught but can be out of touch with the problems of clinical decision making which relate to matters of life and death.

Our study ${ }^{2,3}$ establishes, as Bland agrees, that a high-risk group of infants can be identified at birth. In view of the $63 \%$ greater mortality in the untreated group compared with the treated group can we reasonably withhold increased surveillance from all high-risk infants? We have shown that the balance of probabilities is in favour of surveillance although the difference is not statistically significant ${ }^{4}$. Our health visitors discovered critically ill infants some of whom would almost certainly not have survived but for prompt admission to hospital ${ }^{5}$ and, after surveillance of all high-risk infants, mortality was further reduced to almost exactly the predicted level ${ }^{3}$. In retrospect, the two deaths in the study group could probably have been prevented had our observations been followed by more determined intervention. The weight chart of the first 13-week-old infant showed a marked fall in the rate of growth in the preceding 4 weeks. The other child had been in hospital for failure to thrive and had been discharged too soon.

A substantial proportion of infants have symptoms in the 3 weeks before unexpected death and increased surveillance along the lines of our study could provide active medical care (in preparation). In Finland ${ }^{6}$ and Sweden $^{7}$ the post-perinatal mortality rates were 3.7 and $2.9 \%$ in 1974 compared with 7 per thousand in England and Wales. In both countries more than $98 \%$ of infants are served by child health centres, which average more than eight contacts in Sweden and more than 10 contacts in Finland per child in the first year of life; both rates are apparently much higher than for England and Wales. In France infant mortality has declined since child benefits were made dependent on medical examinations. These observations point to the importance of surveillance $^{8}$. In the light of such evidence we think it would have been unethical to prolong the controlled study in Sheffield ${ }^{4}$.

Our records show that randomisation was suspended only for $8 \mathrm{~d}$ in the Spring of 1973, affecting 16 high-risk infants none of whom died. Between 7 July and 14 September 1974, to avoid overloading the health visitors, only two in five high-risk infants were allocated at random to the study group.

Having advised us to discard some of our data, Bland criticises us for starting a trial with virtually no hope of success. This attack is based on our results, as though we had known what these would be, and ignores our prior information some of which we have reported $^{3}$. Our prior information was that there were between 40 and 50 unexpected infant deaths in Sheffield per year (more than 8,000 births per year; post-perinatal mortality rate $8.5 \%$ 。 giving an unexpected death rate of about $6 \%$ ). The scoring system was planned to include half these deaths in the high-risk group. Our primary object $^{3,9}$, was to obtain prospective data on infants before they died. Surveillance is seldom without effect, so that a highrisk control group was essential both to evaluate the scoring system and to obtain a measure of the effects of the surveillance. We did not anticipate the considerable decline in rates of birth and mortality, the combined effects of 